\section{ORIERTAI COMMUTICATIONS.}

\section{CLINICAL REMARKS ON SYPHILISATION. By JOSEPH SAMPSON GAMGEE.}

Whaterez be the judgment which experience will erentually pronounce upon the merits of syphilisation, there can be no question as to its present claims upon the attention of every one who is interested in lessening the evils of one of the greatest scourges of the human race. Though only a few years have elapsed since the fundamental fact was announced on which the doctrine and practice under consideration are based, it has been repeatedly debated in the medical academies of the continent. Though these have for the most part condemned it, Auzias Turenne in Paris, Sperino in Turin, Boeck in Christiania, and Siegmund in Vienna, persist in its advocacy: though it has been sweepingly condemned as chimerical and immoral, numerous well established facts connected with it await solution, and it has yet to be proved that efforts to lessen the evils of syphilis are contrary to morality. The opportunity which I have recently enjoyed of frequent interviews with Prof. Sperino, privately, and in the Turin Syphilicome, at the head of which be is placed, will, I trust, be deemed a sufficient apology for this communication.

The cases I observed were of old date, the Professor having, for reasons presently to be noticed, suspended his experimental inoculations for the present. In all of them the gradual diminution of the cicatrices from the size of threepenny pieces to pin's points was a striking phenomenon, confirming the doctrine, that in repeated inoculations the system becomes less susceptible of the specific action of the syphilitic virus; or, in other words, the latter gradually loses its power to produce a chancre. Whatever be the clinical value of this fact, I am quite certain it has not yet been accurately determined; its strangeness cannot be exaggerated, particularly when it is considered that, in many cases at any rate, the repeated inoculations exert an injurious influence upon the system; that in some cases certainly the disappearance of secondary symptoms proceeds, pari passu, with the inoculation of chancrous pus, and that in the majority of cases, if not in all, the opinion which one would, $\grave{a}$ priori, be disposed to defend, that the frequency and intensity of the secondary symptoms bear intimate relation to the number, degree, and duration of the primary sores, is demonstratively refuted.

As illustrative of the fact that individuals once syphilised are incapable of contracting the syphilitic virus, Professor Sperino brought under my notice a prostitute who had, notwithstanding uninterrupted assiduity in her vocation, continued free from discase since she was syphilised three years since. Granting that she has probably been exposed on several occasions to the actual danger of infection, her immunity will justly be held to prore little in support of the preventative efficacy of syphilisation by all who reflect upon the not unfrequent historics of dissolute characters of both sexes, who in a long career of vicious indulgence have scarcely been interrupted by even a light clap or a simple excoriated chancre. A large number of cases observed for several years can alone prove the point. According to Professor Sperino, some such evidence is at hand; he thus sums up the cases, proving the curative and prophylactic power of syphilisation.* "Excluding all the cases of incomplete syphilisation, those in whom lesions characteristic of constitutional syphilis supervened, and all those in whom during the two past and in the present year recourse was had to mercurials, and collecting together only those cases, in which there was not manifestation or reproduction of constitutional syphilis, let us see if the body of all these facts be not worthy the attention of those who love the progress of science.

"The indiriduals already affected with primary syphilis,

- Esame critico del rapporto della commissione incaricata dalla R. Acc. Med. Chir. di Torino di studiare la sifilizzezione o state attuale della quistione, per C. Sperino. Torino, 1854, p. 126. and as yet free from constitutional apphilis, axcluding, to be liberal, all doubtful cases, are 44 in number.

"The individuals already affocted with constitutional osphilis, cured with syphilisation alone, in whom the disease has not hitherto re-appeared, are 28.

"The individuals cured of constitutional syphilis with syphilisation and iodide of potassium, in whom the disease has not as yet returned are 4 in number.

" Uniting these 4 to tre 28 preceding, we have 32 cases in which constitutional syphilis disappeared without the aid of mercury, and has not yet re-appeared, and which have been syphilised at the following periods.

1 since 27 months.

\begin{tabular}{|c|c|}
\hline & \\
\hline " & 26 \\
\hline פ & 25 \\
\hline " & 24 \\
\hline " & 23 \\
\hline " & 22 \\
\hline " & 21 \\
\hline " & 18 \\
\hline " & $\begin{array}{l}10 \\
15\end{array}$ \\
\hline
\end{tabular}

1 since $13 \frac{1}{2}$ months.

$1 n 11 n$

10100

$1 " 7 \%$

$3 "$ " 6 "

1305

3

"It is not necessary I should call the attention of the academy to the immense importance of these facts, because they are of themselves sufficient to demonstrate that there is ground for regarding the therapeutic power of syphilisation as radical.

"Being, in the next place, desirous of studying the prophylactic power of syphilisation, I shall only group the cases of completed or partially accomplished syphilisation, practised on prostitutes, they being at every moment exposed to infection.

"There are sixty-fire prostitutes who have not hitherto contracted any new symptom of primary syphilis.

“In other twelve prostitutes, a symptom was observed which left doubts of primary syphilis."

As every one sees, it is still with the support of facts observed by me, that I persist in believing that syphilis is worthy of being studied. The professor not regarding the above imposing facts as conclusive, but merely as warranting further study, has unimpeachable claim upon the support of all, the main springs of whose actions are love of truth and the relief of human suffering.

According to the case of the prostitute above related by me some weight on the positive side of the prophylaxis balance, much greater was that on the negative afforded by two other women, who, since they were syphilised, had contracted sores at the vulva for which they were under Prof. Sperino's treatment during my visit. He denied the sores being chancres, and maintained they were simple excoriations or abrasions from excessive coition. He based this opinion upon the appearance of the sores, and his experience of the incapability of the discharge from sores similar to these, to produce chancres when inoculated. Deeming this opinion too solid to need further experimental evidence, he had dispensed with inoculation in the two cases under consideration. I grant that the sores had not the characters of Hunterian chancres, that they had the appearance of simple sores, and were healing without mercury. But who can positively determine the difference between some venereal and simple sores from appearance alone? It is well known that a considerable number of primary venereal sores have not the Hunterian characters, but present the appearance of simple excoriations, and heal without specific remedies, or without any treatment at all. It must, moreover, be borne in mind that the presumption is in favour of the venereal nature of the sores in question, the origin of which one is, a posterioni, indisposed to explain in a prostitute as the effect of abrasion from excessive coition. As the onus of proof rests on Professor Sperino, no leniency in the shape of a petitio questionis can be allowed to him. He is bound to establish the diagnosis in every case by direct observation, and exclusion of fallacy, so as to remove all doubt. Every suspected sore met with in the prosecution of his studies on syphilisation should be inoculated at different periods of their existence; but even then, if no effect were produced, 
would it be warrantable absolutely to deny the venereal ehsracter of the sore? My thumb would say no ; doubtloesly it was an exceptional case, but, I am quite satisfied from observation, not a solitary one. In the rule, the evidence of inoculation is demonstrative; but whoever reflects how much the progress of knowledge is arrested by the lack of sufficient severity in the observation of individual facts, will see the necessity of exhausting every resource for the exclusion of fallacy in the experimental study of a matter so fraught with difficulty as is the whole subject of venereal disease.

While Professor Sperino's candour, kindness, scientific enthusiasm, and marvellous powers of application, excited my highest admiration and gratitude, love of truth, and anxiety for its attainment, oblige me to confess that I should have been pleased to see him even more then ordinarily severe in his method of clinical study ; nevertheless, the undoubted facts he brought under my notice in his wards, and in published works, abundantly satisfy me that those who have so speedily condemned syphilisation as not only useless but pernicious, have judged prematurely. When it is borne in mind how unjustitiably, bitterly, and vehemently Professor Sperino has been assailed at home and abroad, it is not altogether surprising that he has refused to identify himself with those salamanders who, to use the words of Bishop Hall, are only happy in the fire of contention, and has for the present suspended his experiments: yet, when I consider the vast resources at his disposal, the warranty which his numerous facts supply him with as to the justifiableness of his proceedings, the support he has received from such men as Siegmund, Seutin, and Boeck, I cannot but regret that he should have retracted one step before the ficry declamations of the talented and eloquent, but-be it remembered, in erery question of scientific discovery and merit-jealous Parisians, instead of adopting for his motto the famous lines of the Venusian lyric-

$$
\begin{aligned}
& \text { "Si fractus illabatur orbis } \\
& \text { Impavidum feriunt ruinæ"- }
\end{aligned}
$$

as every one should do who has sufficient confidence in the purity of his intentions and the justifiableness of his means for attaining an object conducive to the welfare of men, however numerous and poignant be the arms with which he is assailed by those who either lack the will or the ability to judge him fairly. Believing as he does that "none of the questions in syphilisation can, in the actual state of science, be definitively solved, and that therefore the new phenomenon is still worthy of accurate study," + I trust Professor Sperino will continue to give to precept the allimportant and powerful succour of example.

In support of my anxiety for further information on this subject, I shall quote the authority of Dr. Boeck, the Professor of Clinical Surgery in the University of Christiania. In a memoir which he published simultaneously in Swedish and French, he remarks: "I believe with $M$. Ricord that it is very doubtful whether constitutional syphilis can be expelled the system when once it has invaded it. Patients have too often been seen, apparently cured, relapse after several years without having exposed themselves to new contagion, or their children come into the world with syphilis; and, as it must be agreed that in mercury we have but an uncertain and deceptive remedy, I have thought it my duty not to hesitate to give trial to a plan which might possibly open to us a new road in the treatment of this disease. The trials which I have made have been conducted without pre-occupation; my only object was to ascertain the merits of syphilisation; therefore I took note of every fact as correctly as my time permitted; and the trials took place under the eyes of students and many of my senior colleagues. To place beyond doubt that I had to deal with syphilitic affections, I caused portraits to be taken of the patients before commencing the treatment; and, in order not to interfere with it, I emplojed no

- As8oclation Medical Jodriral, December 16th, 1853, p. 1108. + Eneme aritico cit p. 141. other remedy, provided this namo be not given to simple unguentum ceratum and a poultice."

In reply to a question from Sperino, as to whether, from "his practical studies of syphilisation, he could conclude that it is worthy of being studied", Professor Boeck thus wrote on the 6th November ult.: * "I can answer by a very positive yes to your question as to whether my practical studies in syphilisation have convinced me that it is worthy of being studied. The first series of my experiments, comprising six persons affected with constitutional syphilis, was attended with such happy results, that I have commenced a second one, consisting of seven individuals similarly affected with constitutional syphilis. As to the conclusions deduced from my first experiments, I only consider three of them important, viz.-

"1. Repeated inoculations produce immunity. Neither friend nor foe can dispute this fact, whose importance in itself is such that it alone would suffice to insure for syphilisation an honourable position.

"2. Nutrition improves by the repeated inoculations.

" 3 . The syphilitic symptoms frequently have a tendency to disappear."

But it must not be overlooked that at least equally eminent authorities have not only condemned syphilisation as useless, but immoral; and on this twofold basis have discountenanced its further study. The question of the morality of syphilsation, so often mooted, and indignantly solved in the negative even by the Parisians, forcibly suggested itself to me as I was roaming about the crowded wards of the Turin Syphilicome, with the image fresh on my mind of the little alley near the Porta Capuara at Naples, thronged by three hundred prostitutes, than which a more revolting and melancholy sight can scarcely be conceived. I am amongst the last who would in the least aggravate the moral or material degradation of these unfortunate women; I am one of the most sanguine in believing that much may be accomplished to alleviate it; but, when I reflect on the enormous ravages of syphilis, or the fact that, in the study of syphilisation, facts have been observed which afford a prospect of those ravages being in part, at any rate, mitigated; that the experimental prosecution of this study does not appear to injure the health of its subjects; that it is impossible to conceive how moral injury can result from endeavouring to cure syphilitic prostitutes by one method rather than by another,-I find no reason to check my appetite for further information on this most interesting subject. But it may be said, if, besides curing these prostitutes, you render them incapable of contracting syphilis; if, besides discovering a more potent remedy for this disease than you now possess, you discover a preventive, you will destroy one of the barriers providentially destined to check illegitimate sexual intercourse. Whilst I have yet to be informed of the evidence in support of this barrier's high mission, while I am quite persuaded it very inadequately fulfils it, and produces far more evil than it appears to do good, I see no sufficient reason against the prosecution of the study of syphilisation. It bcing admitted that we are bound to exert our utmost endearours to cure syphilis, as well as all other liseases, I should like to know upon what principles we are, in the treatment of syphilis, to protest against the real philosophical end of therapeutics-the prevention of disease. Though no one can deny the moral benefits of syphilis are in degrec, at any rate, questionable, that its material evils are numerous and certain, many may question the propriety of persisting in endeavours of problematical efficiency in lessening the latter, and probable tendency to lessening the former. Let Englishmen bear in mind that the chances of moral evil from such endeavours are in the continental syphilicomes reduced to the minimum. Would any Englishman close Ricord's famous Lettres sur la Syphilis in disgust, because trop entrées, for his nationally instinctive, no less than for his professionally acquired notions of literary decorum? or refuse to avail himself of the enormous advantages of the

- Id. p. 183. 
lying-in hospital of this capital, becense apposed not only to Inglish usages, but to all notions of dbeemey ar propriety ? Experience proves the negrative. Much less do I see reason to abste the delight I experience on reflecting that the lowest class of prostitutes, in some of the crowded that the lowest cinstial Europe, are being made the objects capitals of continental kurope, are being mary to preconceived opinions, do not appear to injure them, while they afford a prospect of benefit to the whole family of mankind.

Vienns, 127 Alserrorstadt, July 218t, 1854.

\section{SUCCESSFUL CASE OF EXCISION OF THE KNEE-JOINT.}

By CHARLES COTTON, M.D., F.R.C.S., Senior Surgeon to the West Norfolk and Lynn Hospital.

Thomas NeEPs, aged nine years, son of a peasant, was admitted into the West Norfolk Hospital on April 30th, 1853, with diseased right knee, of three years' standing. There was considerable chronic enlargement of the knee; the limb was semi-flexed; the foot was everted; the tibia was partially displaced backwards; and the patella was drawn upwards and onwards upon the external condyle. The patient had a sickly and worn aspect. He was of ordinary stature, had a sickly and to be spare. Full diet and cod-liver oil were ordered; and he was directed to remain in bed, and to have iodine paint moderately applied to the affected joint.

May 12th. No improvement. The knee was painful and uneasy. Chloroform having been inhaled, the limb was forcibly straightened by grasping the thigh with the left hand, and the leg with the right. This was effected with considerable difficulty (owing to firmly resisting adhesions), but without laceration of the integuments of the ham, which not unusually occurs in old cases of contracted knee. The limb was secured in the extended position upon a M'Intyre's splint; the joint ends of the bones still, however, retaining nearly the same malposition. Flannels, wrung out of hot water, were ordered to be constantly wrung and to be followed by belladonna lotion.

Na 13th. "No increased action or pain in the joint had occurred. He took an anodyne every night.

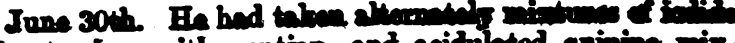

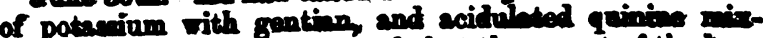
urea. His heelth was improved; but the eapect of the bue was the same, though it was mowe eng thas formedy, enabling him to sit up, and to be mored abont tha wands.

July 9th. The limb was adjusted an a M'Intype oplinto. Made an out-patient, in order that ho might have the benefit of country air.

Aug. 15th. He presented himself, complaining of roturning pain and uneasiness in the kneo. The oplint wan re-adjusted. He was ordered to have cod-liver oil daily, and returned home with an understanding that, should he become worse, he was to re-enter the hospital.

Sept. 27th. Patient readmitted. General health much impaired; was pale and siclly. He had no appetite, got but. little sleep at night, and suffered much from pain in the knoe, which was tender to the touch. The foot, secured to the footpiece, was with the thigh and knee twisted outwards, the petient having turned the splint above to the inner side, giving the whole limb a very awkward appearance. On removing the splint, the integuments were found sound, and the knee much enlarged ; but there did not appear any material fulness from fluid in the joint. The patella was completely dislocated on the outer condyle; the tibia was displaced backwands and outwards; and the condyles of the femur projected forwards and inwards. The whole leg and foot were so greatly rotated outwards on the femur, that the inner ankle was placed directly forwards, whilst the external malleolus was inclined completely in an opposite direction. The least examination of the limb caused apparently agonising pain. The knee was shortly after condemned in consultation; and the choice of amputation or excision being left to the discretion of the surgeon, the latter was determined upon.

Oct. 5th. Operation. The boy, under the complete influence of chloroform, having been placed upon the operating table, the usual letter $\mathbf{H}$ incisions were boldly and quickly made. The flaps having been reflected, the patella being diseased, was at once removed, exposing the joint, and giving exit to a quantity of pus. The remaining fleshy and ligan mentous connexions were further divided by cutting close around the bones; and the partially destroyed crucial ligaments were also detached. The ends of the bones, which were carious and destitute of cartilage, being made to project by

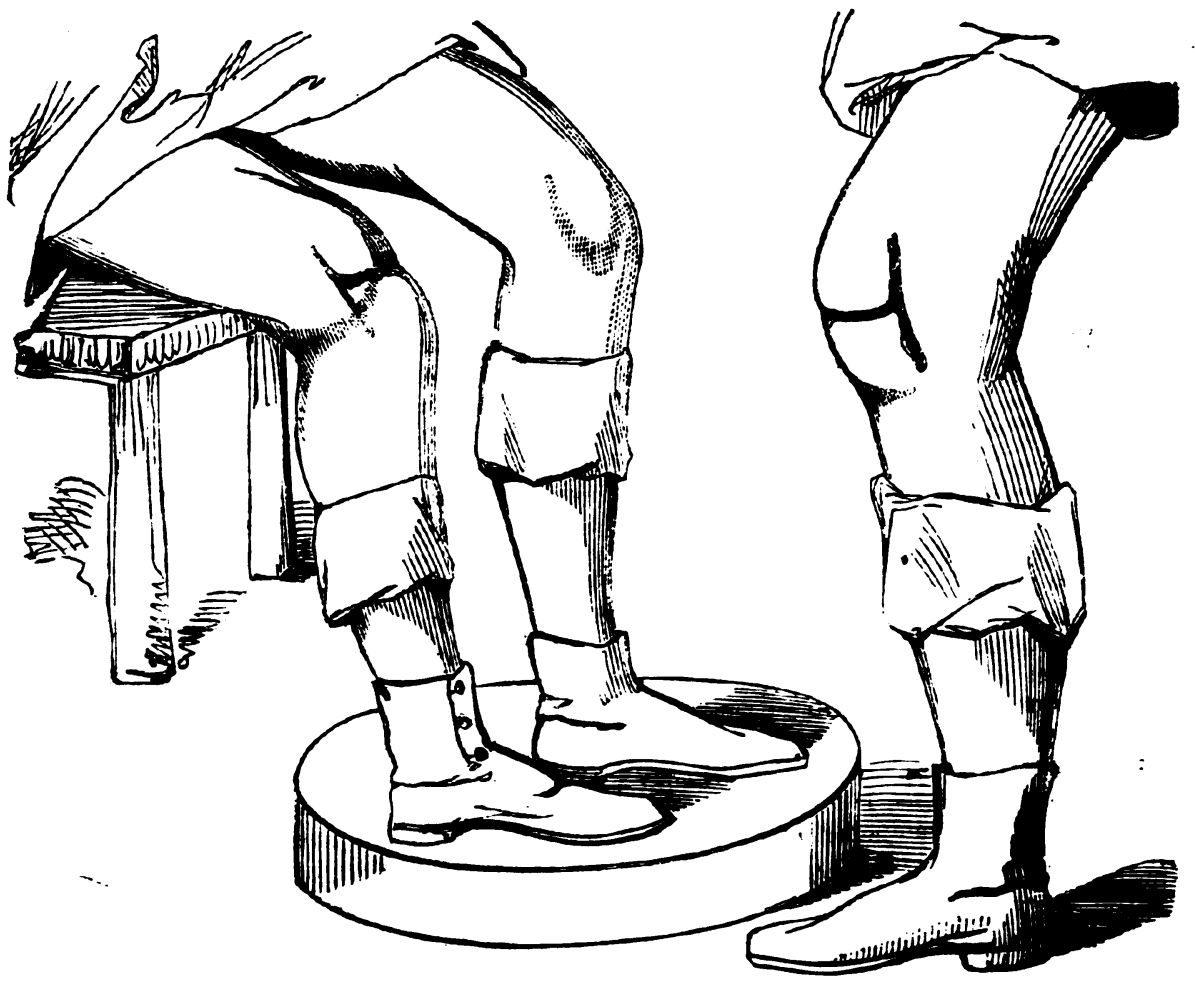

